\title{
ARTICLE
}

Received 18 Mar 2014 | Accepted 24 Jul 2014 | Published 2 Sep 2014

DOI: $10.1038 /$ ncomms5788

\section{Near-field interferometry of a free-falling nanoparticle from a point-like source}

James Bateman ${ }^{1}$, Stefan Nimmrichter ${ }^{2}$, Klaus Hornberger ${ }^{2} \&$ Hendrik Ulbricht $^{1}$

Matter-wave interferometry performed with massive objects elucidates their wave nature and thus tests the quantum superposition principle at large scales. Whereas standard quantum theory places no limit on particle size, alternative, yet untested theories-conceived to explain the apparent quantum to classical transition-forbid macroscopic superpositions. Here we propose an interferometer with a levitated, optically cooled and then free-falling silicon nanoparticle in the mass range of one million atomic mass units, delocalized over $>150 \mathrm{~nm}$. The scheme employs the near-field Talbot effect with a single standing-wave laser pulse as a phase grating. Our analysis, which accounts for all relevant sources of decoherence, indicates that this is a viable route towards macroscopic high-mass superpositions using available technology.

\footnotetext{
${ }^{1}$ Physics and Astronomy, University of Southampton, Highfield SO17 1BJ, UK. ${ }^{2}$ Fakultät für Physik, Universität Duisburg-Essen, 47048 Duisburg, Germany. Correspondence and requests for materials should be addressed to H.U. (email: h.ulbricht@soton.ac.uk).
} 
$\mathrm{M}$ atter-wave interference with particles of increasing size and mass is a natural and viable method for testing the validity of the quantum superposition principle at unprecedented macroscopic scales ${ }^{1-4}$. Macroscopic path separations are nowadays routinely achieved in atom interferometry ${ }^{5-7}$, and technological advances in the control of opto-mechanical systems ${ }^{8}$ promise that much more massive objects may be delocalized ${ }^{9-13}$, albeit with spatial separations smaller than a single atom.

Recent proposals put forward nanoparticle interferometry ${ }^{14,15}$ in the mass range of $10^{6}-10^{9}$ AMU to surpass the mass records currently held by molecule diffraction experiments ${ }^{3,16}$, while maintaining spatial separations large enough to be resolved by optical means. A first demonstration with molecular clusters ${ }^{15}$ is still far away from the mentioned high-mass regime due to difficult experimental challenges, mainly concerning the source and detection. The realization of a proposed double-slit scheme with silica nanospheres ${ }^{14}$ requires motional ground-state cooling, which is an equally challenging task.

Quite recently, optical feedback cooling has been demonstrated for 100-nm-sized particles ${ }^{17,18}$, based on pioneering work that demonstrated the trapping of polystyrene and glass microspheres ${ }^{19}$, trapping of viruses and bacteria ${ }^{20}$ and even of complete cells ${ }^{21}$ in solutions and high vacuum. Cavity cooling of particles of similar size was proposed ${ }^{22}$ and recently achieved ${ }^{23,24}$ in one dimension, with temperatures in the milli-Kelvin range. Although this is still far above the ground state of a typical $100-\mathrm{kHz}$ trap, we will argue that high-mass interference can be realized experimentally with motional temperatures already achieved by optical cooling.

In this paper, we present a near-field interference scheme for $10^{6} \mathrm{AmU}$ particles. It is based on the single-source Talbot effect ${ }^{25}$ due to a single optical phase grating, as opposed to the threegrating scenario in Talbot-Lau interference experiments ${ }^{3}$. Optically trapped silicon nanospheres, feedback-stabilized to a thermal state of about $20 \mathrm{mK}$, provide a sufficiently coherent source. Individual particles are dropped and diffracted by a standing UV laser wave, such that interference of neighbouring diffraction orders produces a resonant near-field fringe pattern. In order to record the interferogram, the nanospheres are deposited on a glass slide and their arrival positions are recorded via optical microscopy. We argue that the choice of silicon, due to its specific material characteristics, will yield reliable high-mass interference, unaffected by environmental decoherence, in a set-up that can be realized with present-day technology.

\section{Results}

Proposed experiment. The proposed scheme is sketched in Fig. 1. In the first stage of the experiment, a silicon particle is captured in an optical dipole trap by a lens system of numerical aperture 0.8 focusing a $1,550 \mathrm{~nm}$ laser to a waist of $860 \mathrm{~nm}$ (ref. 26); the interaction of nanoparticles with light is described further in Supplementary Note 1. The trapping light is collected and used to determine the position of the particle ${ }^{18}$, which is feedback cooled over many trapping cycles to about $T=20 \mathrm{mK}$ of mean translational energy along the horizontal $x$-axis, implying a momentum uncertainty $\sigma_{p}=\sqrt{m k_{\mathrm{B}} T}$ of about $\sigma_{p} / m=1.2 \mathrm{~cm} \mathrm{~s}^{-1}$. A laser power of $55 \mathrm{~mW}$ results in a trap frequency of $v_{\mathrm{M}}=200 \mathrm{kHz}$ and a position uncertainty $\sigma_{x}$ $<10 \mathrm{~nm}$; see Supplementary Note 2 . The trap thus serves as a nearly point-like matter-wave source for diffraction.

After feedback cooling, the particle is released from the trap and falls for $t_{1}=160 \mathrm{~ms}$ before it is illuminated by a frequencytrippled Nd:YAG laser pulse at $355 \mathrm{~nm}$ with a pulse length of $10 \mathrm{~ns}$ and an energy $E_{\mathrm{G}} \leq 500 \mu \mathrm{J}$. The pulse is retro-reflected by a a

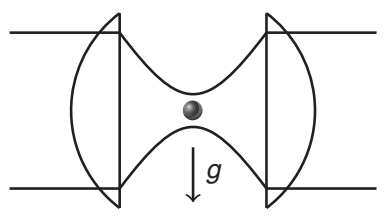

$125 \mathrm{~mm}$

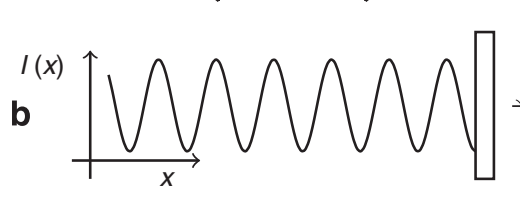

$275 \mathrm{~mm}$

C

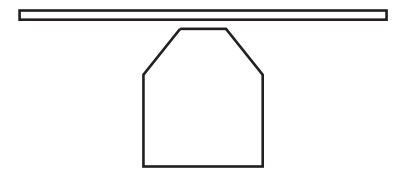

Figure 1 | Schematic of the proposed experiment. (a) Laser light at $1,550 \mathrm{~nm}$ creates a harmonic trap for single-silicon nanospheres. After feedback cooling to $20 \mathrm{mK}$, the particle is released and falls for $125 \mathrm{~mm}$, where it passes a phase grating (b) provided by a retro-reflected nanosecond pulse at $355 \mathrm{~nm}$. $275 \mathrm{~mm}$ further down the particle is adsorbed on a glass slide (c), where the arrival position is recorded with $100 \mathrm{~nm}$ accuracy via optical microscopy (d).

mirror to form a standing-wave phase grating with period $d=\lambda_{\mathrm{G}} / 2$, which diffracts the particle by modulating the matterwave phase through the dipole interaction. The Talbot time, which sets the scale for near-field interference ${ }^{3}$, is thus given by $t_{\mathrm{T}}=m d^{2} / h \approx 80 \mathrm{~ms}$. The laser beam must be expanded such that the waist is larger than the uncertainty in position $\sigma_{p} t_{1} / m \approx 2 \mathrm{~mm}$ accrued during free flight. Moreover, the orientation of the grating must be angularly stable to less than micro-radians to avoid blurring of the interferogram due to acceleration of the particle under gravity, and positionally stable to within $30 \mathrm{~nm}$ relative to the initial particle position; see Supplementary Note 3.

After the grating, the particle undergoes free fall for $t_{2}=126 \mathrm{~ms}$, forming an interference pattern when it arrives on the glass slide. The arrival position can be detected by absorption imaging with visible light. Fitting to the known point-spread function of the imaging system permits $100 \mathrm{~nm}$ positional accuracy ${ }^{27}$; see Supplementary Note 4 . The density pattern depicted in Fig. $2 \mathrm{a}$ is predicted to appear after many runs of the experiment. In the following, we discuss the theoretical description of the interference effect and the experimental constraints.

Theoretical model. Our starting point for evaluating the interference effect is the trapped thermal state of motion, a Gaussian mixture with standard deviations $\sigma_{x}=\sqrt{k_{\mathrm{B}} T / 4 \pi^{2} m v_{\mathrm{M}}^{2}}$ and $\sigma_{p}=\sqrt{m k_{\mathrm{B}} T}$. The particle will be illuminated by a uniform standing-wave pulse oriented along the horizontal $x$-axis (see Fig. 1), so that the $y$ - and $z$-motion can be ignored.

The near-field diffraction effect including all relevant decoherence mechanisms is best captured in a quantum phasespace description $^{28}$. For the present purposes it is most useful to work with the characteristic function representation $\chi(s, q)$, that is, the Fourier transform of the Wigner function ${ }^{29}$ of a given quantum state $\rho$. Here, we summarize the detailed derivation given in Supplementary Methods. The initial Gaussian state,

$$
\chi_{0}(s, q)=\exp \left(-\frac{\sigma_{x}^{2} q^{2}+\sigma_{p}^{2} s^{2}}{2 \hbar^{2}}\right)
$$



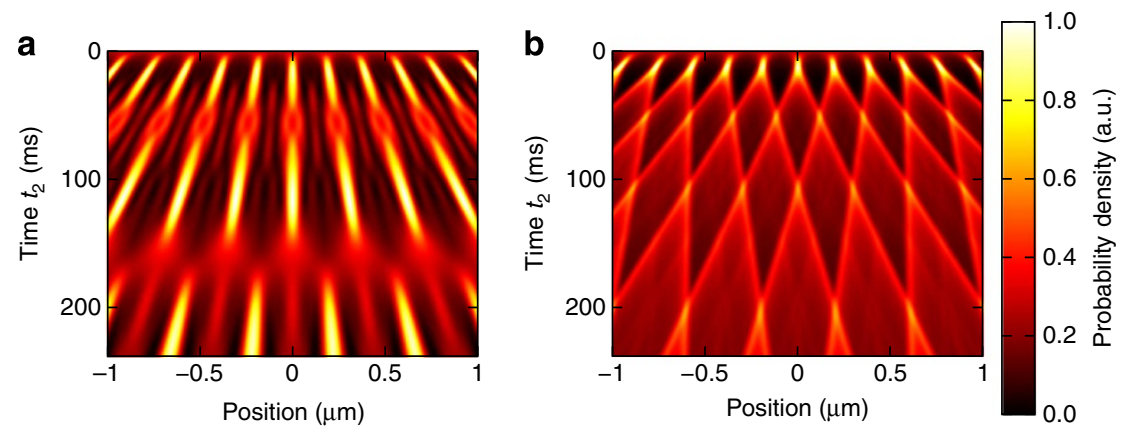

Figure 2 | Simulated fringe patterns below the grating. (a) Quantum and (b) classical probability densities at different times $t_{2}$ after the grating pulse. We assume the width of the trap state to be $\sigma_{x}=10 \mathrm{~nm}$ and a time of flight to the grating of $t_{1}=160 \mathrm{~ms}$; the grating has the period $d=177 \mathrm{~nm}$ with the maximal phase modulation set to $\phi_{0}=\pi$. The interference pattern is detected after $t_{2}=126 \mathrm{~ms}$ in the set-up of Fig. 1. Quantum mechanics then predicts high-contrast fringes, which cannot be explained classically.

first evolves freely for a time $t_{1}, \chi_{1}(s, q)=\chi_{0}\left(s-q t_{1} / m, q\right)$, before it is illuminated by the optical grating pulse of period $d$. Given an almost point-like initial spread $\sigma_{x} / d \ll 1$, the matter waves must evolve for at least the Talbot time $t_{\mathrm{T}}$ to ensure that they are delocalized over adjacent grating nodes in order to be able to interfere. The initial momentum, on the other hand, is spread over many grating momenta, $\sigma_{p} d / h \gg 1$, so that the time-evolved state extends over many grating periods. That is, if particles are only detected in a finite detection window around the centre of the distribution in the end, we can neglect the Gaussian density profile by writing

$$
\chi_{1}(s, q) \approx \frac{\sqrt{2 \pi} \hbar}{\sigma_{p}} \exp \left(-\frac{\sigma_{x}^{2} q^{2}}{2 \hbar^{2}}\right) \delta\left(s-\frac{q t_{1}}{m}\right) .
$$

The particle interacts with the standing-wave pulse through its optical polarizability $\alpha=4 \pi \varepsilon_{0} R^{3}\left(n_{\mathrm{Si}}^{2}-1\right) /\left(n_{\mathrm{Si}}^{2}+2\right)$, determined by the particle radius $R$ and its complex refractive index $n_{\mathrm{Si}}$ at the grating wavelength $\lambda_{\mathrm{G}}=2 d$. In the limit of short pulse durations $\tau$, this imprints the phase $\phi(x)=\phi_{0} \cos ^{2}(\pi x / d)$ on the matterwave state ${ }^{5}$, where $\phi_{0}=2 \operatorname{Re}(\alpha) E_{\mathrm{G}} / \hbar c \varepsilon_{0} a_{\mathrm{G}}$ depends on the energy $E_{\mathrm{G}}$ and spot area $a_{\mathrm{G}}$ of the pulse. The characteristic function transforms as $\chi_{1}(s, q) \rightarrow \sum B_{n}(s / d) \chi_{1}(s, q+n h / d)$, where the $B_{n}$ are Talbot coefficients, given in terms of Bessel functions ${ }^{30}$,

$$
B_{n}(\xi)=J_{n}\left(\phi_{0} \sin \pi \xi\right) \text {. }
$$

Incoherent effects due to absorption or scattering of laser photons are negligible for the nanoparticles considered here (Supplementary Methods); nevertheless, our numerical simulations include both effects.

The final density distribution $w(x)=\langle x|\rho| x\rangle$, that is, the probability to find the particle at position $x$ after another free time evolution by $t_{2}$, then takes the form

$$
\begin{aligned}
w(x) & =\frac{m}{\sqrt{2 \pi} \sigma_{p}\left(t_{1}+t_{2}\right)} \sum_{n} B_{n}\left[\frac{n t_{1} t_{2}}{t_{\mathrm{T}}\left(t_{1}+t_{2}\right)}\right] \\
& \times \exp \left[\frac{2 \pi i n x}{D}-\frac{2 \pi^{2} n^{2} \sigma_{x}^{2} t_{2}^{2}}{d^{2}\left(t_{1}+t_{2}\right)^{2}}\right] .
\end{aligned}
$$

It describes a periodic fringe pattern oscillating at the geometrically magnified grating period $D=d\left(t_{1}+t_{2}\right) / t_{1}$ (ref. 25). The fringe amplitudes, given by the Talbot coefficients (3), are diminished the larger the spread $\sigma_{x}$ of the initial state (1).

An exemplary density pattern (4) is plotted in Fig. 2a for varying time $t_{2}$. The simulation was performed for $10^{6} \mathrm{AMU}$ silicon particles, assuming realistic experimental parameters and including the influence of environmental decoherence. It shows pronounced interference fringes with visibilities of up to $75 \%$.

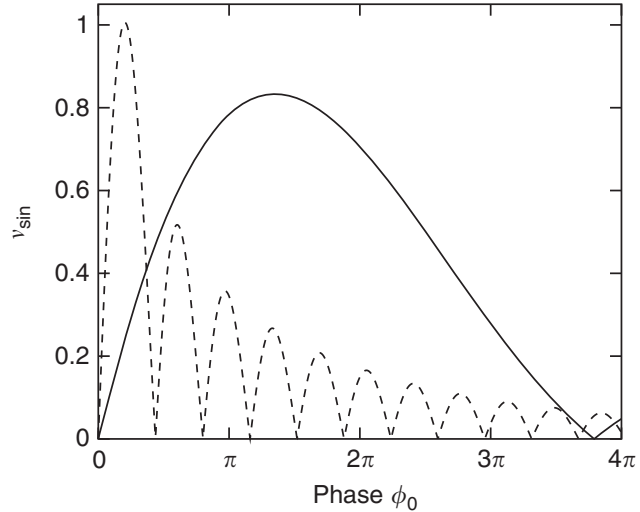

Figure 3 | Expected quantum (solid) and classical (dashed) sinusoidal visibilities as a function of the phase-modulation parameter $\phi_{0}$. This dependence on the pulse energy illustrates clearly the difference between the predictions.

The pattern in Fig. $2 b$ is the result of a classical simulation assuming that the particles are moving on ballistic trajectories. A lensing effect due to the strong dipole forces exerted by the standing-wave field is here responsible for the density modulation. This classical result is obtained simply by replacing $\sin \pi \xi$ by $\pi \xi$ in expression (3) for the grating coefficient ${ }^{30}$.

The clear difference between the quantum and the classical pattern is captured by the sinusoidal fringe visibility, the ratio between the amplitude and the offset of a sine curve of period $D$ fitted to the density pattern (4):

$$
\mathcal{V}_{\text {sin }}=2\left|B_{1}\left[\frac{t_{1} t_{2}}{t_{\mathrm{T}}\left(t_{1}+t_{2}\right)}\right]\right| \exp \left[-\frac{2 \pi^{2} \sigma_{x}^{2} t_{2}^{2}}{d^{2}\left(t_{1}+t_{2}\right)^{2}}\right] .
$$

As shown in Fig. 3, the classical and the quantum prediction differ significantly: the classical theory predicts many regions of low contrast as a function of $\phi_{0}$, whereas the quantum prediction exhibits a slow $\phi_{0}$ dependence. The highest quantum visibility amounts to $83 \%$ at $\phi_{0}=1.4 \pi$.

Accounting for decoherence. A realistic assessment of the proposed scheme must also include the influence of collisional and thermal decoherence ${ }^{28}$. This is incorporated into (4) by multiplying each Fourier component with a reduction factor of the form

$$
R_{n}=\exp \left\{-\Gamma\left[1-f\left(\frac{n h t_{2}}{m D}\right)\right]\left(t_{1}+t_{2}\right)\right\}
$$


where $\Gamma$ gives the rate and $f(x)$ determines the spatial resolution of decoherence events of a certain class. In our simulation we accounted for collisions with residual gas particles, scattering and absorption of blackbody photons, and thermal emission of radiation using a realistic microscopic description. Each process contributes another factor $R_{n}$ listed in the Supplementary Methods; the rate of thermal emission depends on time since the particle loses internal energy and cools during flight.

Experimental constraints. As a major concern for the successful implementation of the experiment, environmental decoherence must be kept sufficiently low. According to our simulations, collisional decoherence can be essentially avoided at ultra-high vacuum pressures of $10^{-10}$ mbar.

Radiative decoherence is suppressed by choosing silicon spheres because they are essentially transparent at typical wavelengths of room temperature blackbody radiation. The thermal emission of photons is determined by the internal temperature of the nanospheres, which is set in the trapping stage of the experiment. A trapping intensity of $90 \mathrm{~mW} \mu \mathrm{m}^{-2}$ leads to an initial heating rate $\partial_{t} T_{\text {int }}=200 \mathrm{~K} \mathrm{~s}^{-1}$ and an equilibrium temperature of $1,600 \mathrm{~K}$. This high value is a consequence of the low blackbody emissivity of silicon ${ }^{31}$, implying that the particle does not lose heat efficiently while in the trap. Nevertheless, due to the high refractive index $n_{\mathrm{Si}}=3.48$ of silicon, the particle may be trapped for well in excess of a second before the temperature rises that high. This time corresponds to about $10^{5}$ trap oscillations, a sufficient period to perform parametric feedback cooling of the motion to $T=20 \mathrm{mK}$; see Supplementary Note 5 . The low emissivity of silicon is the essential advantage compared to other materials such as silica, for which much work in this field has been done ${ }^{10,11,23}$. We find that to perform this experiment with silica would require cryogenic cooling of both apparatus and nanoparticle to $100 \mathrm{~K}$, whereas thermal decoherence of silicon becomes important only at internal temperatures in excess of $1,000 \mathrm{~K}$. Moreover, the high refractive index of silicon compared to the value $n_{\mathrm{SiO} 2}=1.44$ of silica means that less optical power is required to trap the sphere and to monitor its position ${ }^{24}$.

As an additional advantage, silicon absorbs strongly at optical frequencies, which simplifies the detection of the interferogram. In principle, this would also affect the interaction with the grating laser, since a particle at the anti-node of the grating absorbs on average $n_{0}=0.12 \phi_{0}$ photons. For a grating laser waist of $30 \mathrm{~mm}$ we anticipate a phase modulation of $\phi_{0} / E_{\mathrm{G}}=50 \mathrm{rad} \mathrm{mJ}^{-1}$ and hence we can access $\phi_{0} \leq 4 \pi$. The finite absorption of grating photons, which is included in the simulations, disturbs the interferogram little.

\section{Discussion}

We presented a viable scheme for high-mass nanoparticle interferometry, which employs only a single optical diffraction element and requires only moderate motional cooling. The set-up would operate in ultra-high vacuum at room temperature. It is limited to masses up to $10^{6} \mathrm{AMU}$ mainly by the growing Talbot time and free-fall distance ${ }^{32}$. Interferometry in a microgravity environment could pave the way to even higher masses ${ }^{33}$.

Remarkably, with path separations of up to $150 \mathrm{~nm}$ and interrogation times of $300 \mathrm{~ms}$, the presented scheme is already sensitive to alternative theories beyond the Schrödinger equation. The renowned collapse model of continuous spontaneous localization (CSL) ${ }^{34}$ could be probed in its current formulation ${ }^{4}$. In fact, a successful demonstration of interference with a visibility exceeding $42 \%$ would bound the localization rate to $\lambda_{\mathrm{CSL}}<1.4 \times 10^{-11} \mathrm{~Hz}$, a value at the lower end of recent estimates for this parameter ${ }^{35,36}$; see Supplementary Discussion.
Such a superposition experiment can be associated with a macroscopicity value of $\mu=18$ (ref. 37), substantially exceeding that of every present-day matter-wave experiment and comparing well with the most ambitious micromirror superposition proposals ${ }^{9}$.

\section{References}

1. Clauser, J. in Experimental Metaphysics (eds Cohen, R. S., Horne, M. \& Stachel, J.) 1-11 (Kluwer Academic, 1997).

2. Leggett, A. J. Testing the limits of quantum mechanics: motivation,state of play, prospects. J. Phys.: Condens. Matter 14, R415 (2002).

3. Hornberger, K., Gerlich, S., Haslinger, P., Nimmrichter, S. \& Arndt, M. Colloquium: quantum interference of clusters and molecules. Rev. Mod. Phys. 84, 157 (2012).

4. Bassi, A., Lochan, K., Satin, S., Singh, T. P. \& Ulbricht, H. Models of wave-function collapse, underlying theories, and experimental tests. Rev. Mod. Phys. 85, 471-527 (2013).

5. Müller, H., Chiow, S.-w., Long, Q., Herrmann, S. \& Chu, S. Atom interferometry with up to 24-photon-momentum-transfer beam splitters. Phys. Rev. Lett. 100, 180405 (2008).

6. Müntinga, H. et al. Interferometry with Bose-Einstein condensates in microgravity. Phys. Rev. Lett. 110, 093602 (2013).

7. Dickerson, S. M., Hogan, J. M., Sugarbaker, A., Johnson, D. M. S. \& Kasevich, M. A. Multiaxis inertial sensing with long-time point source atom interferometry. Phys. Rev. Lett. 111, 083001 (2013).

8. Aspelmeyer, M., Kippenberg, T. J. \& Marquardt, F. Cavity Optomechanics. Preprint at arXiv:1303.0733 (2013).

9. Marshall, W., Simon, C., Penrose, R. \& Bouwmeester, D. Towards quantum superpositions of a mirror. Phys. Rev. Lett. 91, 130401 (2003).

10. Chang, D. et al. Cavity opto-mechanics using an optically levitated nanosphere. Proc. Natl Acad. Sci. USA 107, 1005-1010 (2010).

11. Romero-Isart, O., Juan, M. L., Quidant, R. \& Cirac, J. I. Toward quantum superposition of living organisms. New J. Phys. 12, 033015 (2010).

12. Scala, M., Kim, M. S., Morley, G. W., Barker, P. F. \& Bose, S. Matter-wave interferometry of a levitated thermal nano-oscillator induced and probed by a spin. Phys. Rev. Lett. 111, 180403 (2013).

13. Yin, Z.-q., Li, T., Zhang, X. \& Duan, L. Large quantum superpositions of a levitated nanodiamond through spin-optomechanical coupling. Phys. Rev. A 88, 033614 (2013).

14. Romero-Isart, O. et al. Large quantum superpositions and interference of massive nanometer-sized objects. Phys. Rev. Lett. 107, 020405 (2011).

15. Nimmrichter, S., Haslinger, P., Hornberger, K. \& Arndt, M. Concept of an ionizing time-domain matter-wave interferometer. New J. Phys. 13, 075002 (2011).

16. Eibenberger, S., Gerlich, S., Arndt, M., Mayor, M. \& Tüxen, J. Matter-wave interference with particles selected from a molecular library with masses exceeding 10,000 amu. Phys. Chem. Chem. Phys. 15, 14696-14700 (2013).

17. Li, T., Kheifets, S. \& Raizen, M. Millikelvin cooling of an optically trapped microsphere in vacuum. Nat. Phys. 7, 527-530 (2011).

18. Gieseler, J., Deutsch, B., Quidant, R. \& Novotny, L. Subkelvin parametric feedback cooling of a laser-trapped nanoparticle. Phys. Rev. Lett. 109, 103603 (2012).

19. Ashkin, A., Dziedzic, J., Bjorkholm, J. \& Chu, S. Observation of a single-beam gradient force optical trap for dielectric particles. Opt. Lett. 11, 288-290 (1986)

20. Ashkin, A. \& Dziedzic, J. Optical trapping and manipulation of viruses and bacteria. Science 235, 1517-1520 (1987).

21. Ashkin, A., Dziedzic, J. \& Yamane, T. Optical trapping and manipulation of single cells using infrared laser beams. Nature 330, 769-771 (1987).

22. Barker, P. Doppler cooling a microsphere. Phys. Rev. Lett. 105, 073002 (2010).

23. Kiesel, N. et al. Cavity cooling of an optically levitated nanoparticle. Proc. Natl Acad. Sci. USA 110, 14180-14185 (2013).

24. Asenbaum, P., Kuhn, S., Nimmrichter, S., Sezer, U. \& Arndt, M. Cavity cooling of free silicon nanoparticles in high vacuum. Nat. Commun. 4, 2743 (2013).

25. Brezger, B., Arndt, M. \& Zeilinger, A. Concepts for near-field interferometers with large molecules. J. Opt. B. 5, S82 (2003).

26. Richards, B. \& Wolf, E. Electromagnetic diffraction in optical systems. II. Structure of the image field in an aplanatic system. Proc. R. Soc. Lond. A 253, 358-379 (1959).

27. Hell, S. W. Far-field optical nanoscopy. Science 316, 1153 (2007).

28. Hornberger, K., Sipe, J. \& Arndt, M. Theory of decoherence in a matter wave Talbot-Lau interferometer. Phys. Rev. A 70, 053608 (2004).

29. Schleich, W. P. Quantum Optics in Phase Space (Wiley-VCH, 2001).

30. Hornberger, K. et al. Theory and experimental verification of Kapitza-DiracTalbot-Lau interferometry. New J. Phys. 11, 043032 (2009).

31. Palik, E. D. \& Ghosh, G. Handbook of Optical Constants of Solids: Five-Volume Set (Academic Press, 1998). 
32. Nimmrichter, S., Hornberger, K., Haslinger, P. \& Arndt, M. Testing spontaneous localization theories with matter-wave interferometry. Phys. Rev. A 83, 043621 (2011).

33. Kaltenbaek, R. et al. Macroscopic quantum resonators (MAQRO). Exp. Astron. 34, 123-164 (2012).

34. Ghirardi, G. C., Pearle, P. \& Rimini, A. Markov processes in Hilbert space and continuous spontaneous localization of systems of identical particles. Phys. Rev. A 42, 78 (1990).

35. Adler, S. L. Lower and upper bounds on CSL parameters from latent image formation and IGM heating. J. Phys. A 40, 2935 (2007).

36. Bassi, A., Deckert, D.-A. \& Ferialdi, L. Breaking quantum linearity: constraints from human perception and cosmological implications. Europhys. Lett. 92, 50006 (2010).

37. Nimmrichter, S. \& Hornberger, K. Macroscopicity of mechanical quantum superposition states. Phys. Rev. Lett. 110, 160403 (2013).

\section{Acknowledgements}

Funding by the EPSRC (EP/J014664/1), the Foundational Questions Institute (FQXi) through a Large Grant, and by the John F Templeton foundation (grant 39530) is gratefully acknowledged. This work was also partially supported by the European Commission within NANOQUESTFIT (No. 304886).

\section{Author contributions}

H.U. conceived the interferometer and initiated the research. J.B. and S.N. designed the scheme and performed calculations and simulations. K.H. advised on the theory All authors discussed the results and wrote the manuscript.

\section{Additional information}

Supplementary Information accompanies this paper at http://www.nature.com/ naturecommunications

Competing financial interests: The authors declare no competing financial interests.

Reprints and permission information is available online at http://npg.nature.com/ reprintsandpermissions/

How to cite this article: Bateman, J. et al. Near-field interferometry of a free-falling nanoparticle from a point-like source. Nat. Commun. 5:4788 doi: 10.1038/ncomms5788 (2014). 